\title{
Sobre la mejora humana por medio de las tecnologías convergentes*
}

\author{
José Manuel de Cózar Escalante**
}

\begin{abstract}
ResUMEN: Un conjunto de tecnologías están convergiendo en su aplicación a la mejora del cuerpo y de la mente del ser humano. Se trata de la combinación de, entre otras, la nanotecnología, las tecnologías de la información y de la comunicación, la biotecnología, la biología sintética, la neurociencia, la neurotecnología, la robótica y la inteligencia artificial. Las aplicaciones ya disponibles, o previstas para un futuro más o menos cercano, están suscitando un notable debate ético a nivel internacional. En este artículo se plantean los aspectos principales de dicha controversia y se sugiere la necesidad de una evaluación ética temprana de los programas de mejora humana.

PALABRAS CLAVE: mejora humana, tecnologías convergentes, evaluación ética.
\end{abstract}

AвSTRACT: On human enhancement by means of converging technologies.

A set of technologies are converging in their application to improve the human body and mind. The combination includes nanotechnology, information technology and communications, biotechnology, synthetic biology, cognitive science, neurotechnology, robotics and artificial intelligence. Applications that are already available or those planned for the near future are attracting considerable international ethical debate. This paper discusses the main aspects of the controversy and suggests the need for an early ethical assessment of human enhancement programs.

KEYWORDs: human enhancement, converging technologies, ethical assessment.

\section{INTRODUCCIÓN}

Asistimos, a nivel mundial, a un renovado debate sobre la legitimidad de emplear la tecnología para mejorar las capacidades físicas y mentales de los individuos más allá de los contextos estrictamente terapéuticos (lo que en inglés se conoce como "human enhancement"). Por ejemplo, en el deporte profesional, en los últimos años, se ha producido una escalada de los escándalos por dopaje. Desde hace algún tiempo la utilización de las terapias génicas para mejorar el rendimiento de los atletas (dopaje genético) constituye un secreto a voces que a duras penas, y sólo desde muy recientemente, puede ser detectado mediante controles realizados al efecto. Otro caso muy debatido es el de la estimulación cerebral profunda. Se trata de una técnica de implante cerebral usada desde hace años para tratar los síntomas del Parkinson y de otros desórdenes neurológicos. Puede igualmente aliviar la depresión severa. Hasta

\footnotetext{
* Este trabajo se enmarca dentro del proyecto de investigación "Evaluación del proceso de transferencia de nuevos materiales nanotecnológicos en equipos de diagnóstico y tratamiento médico" (EvalnanomedC200801000076), financiado por la Agencia Canaria de Investigación, Innovación y Sociedad de la Información y el Fondo Europeo de Desarrollo Regional (FEDER).

** Doctor en filosofía por la Universidad de Valencia (España). Profesor titular de la Universidad de La Laguna, Tenerife. Coordinador del grupo de investigación social en nanotecnología (GRISON), formado por investigadores españoles. Facultad de Filosofía, Campus de Guajara, 38071 La Laguna, Tenerife, España, Teléfono: +34 922317878, Fax: +34 922317879, Correo electrónico: jcozar@ull.es
} 
aquí, nos movemos en el terreno de la terapia. Sin embargo, esta técnica y otras no invasivas más recientes, destinadas al tratamiento de diversos trastornos neuropsiquiátricos (como la estimulación magnética transcraneal) pueden mejorar el buen humor y el bienestar psicológico de individuos considerados sanos y provocar otras alteraciones psicológicas que supuestamente supondrían una mejora con respecto a los estados normales: expresado de una manera un tanto sensacionalista, permitirían presionar una especie de "botón de la felicidad", términos con los que el hallazgo fue presentado en algunos medios de comunicación (EGE, 2005).

En un sentido laxo, herramientas y tecnologías para mejorar al ser humano en un aspecto u otro se vienen usando desde siempre: herramientas para aumentar su potencia y facilitar el trabajo físico, transportes para desplazarse más rápido, uso del fuego, de las técnicas agrícolas y de los diversos procedimientos culinarios para alimentarlo mejor, libros y otros soportes para codificar y transmitir informaciones, materiales pedagógicos para facilitar el aprendizaje y formar las mentes, o medicinas para mejorar el estado de salud, entre otros innumerables ejemplos. De una forma o de otra, esas ayudas técnicas han ido aparejadas al sueño humano, a lo largo de la historia, de superar sus limitaciones físicas y mentales. Ahora bien, lo que se debate encendidamente aquí, con posturas a menudo muy encontradas, es el derecho a operar cambios continuados, radicales y tal vez irreversibles en el cuerpo y la mente humanas para percibir con más precisión nuestro entorno, hacernos más fuertes y resistentes, y muchas otras "mejoras", bien mediante la implantación de dispositivos, bien mediante la alteración orgánica o genética del cuerpo. Incluso modificar nuestra especie, todo ello con objeto de vencer de una vez por todas nuestras "imperfecciones" naturales, los condicionantes impuestos por la evolución biológica (o por Dios, para los creyentes).

La nanotecnología puede ser aplicada en algunas tecnologías de mejora humana, que lindan con la nanomedicina, tales como las que se orientan a la prevención sanitaria y a la regeneración de tejidos y órganos. Otras aplicaciones son más banales -al menos a primera vista-, como la posibilidad de realizar tatuajes que cambien a voluntad de su portador, o que le sirvan para interactuar con su entorno (marcación, visionado de datos, etc.). Una de las posibilidades que más se discute sería la colocación de "implantes neuronales" en el cerebro para acceder a la información y procesarla de manera más rápida y eficiente. El poder de las nanotecnologías aumenta por su convergencia con otras tecnologías, lo que se conoce por convergencia NBIC: Nano (nanotecnología), BIO (biotecnología, biología sintética), INFO (tecnologías de la información y de la comunicación) y COGNO (ciencia cognitiva, neurociencia, neurotecnología). A éstas cabe añadir la robótica y la inteligencia artificial, entre otras.

Los temas del debate filosófico y ético sobre la mejora humana mediante el conjunto de las tecnologías convergentes son, principalmente: ${ }^{1}$

- riesgos para la salud, la privacidad y la seguridad,

- usos militares (soldados "modificados"),

- derecho a elegir libremente vs. limitación de la libertad,

- modificaciones del concepto de salud y de enfermedad,

1 Véanse, entre otros, Ach y Lüttenberg, (2008); Allhoff et al., (2009); Coenen et al. (2009). Una discusión centrada específicamente en los impactos éticosociales de la nanotecnología puede hallarse en Cózar de (2009 y 2010). 
- implicaciones para la autopercepción del ser humano (dignidad e integridad humanas, "condición humana")

- consecuencias negativas para las instituciones sociales;

- injusticia social (división entre los "mejorados" y los "no mejorados").

\section{SObRe LA PROBLEMÁtiCA DEFINICIÓN DE “MEJORA hUMANA”}

Tanto en la teoría como en la práctica es complicado especificar con exactitud qué se quiere decir con mejora humana. Ello es debido a que la noción de mejora implica mejorar a los seres humanos más allá de su estado normal, físico o mental. En un sentido algo más técnico, se trataría de llevar nuestras capacidades más allá del rango de funcionamiento típico de la especie o de lo estadísticamente normal. Ahora bien, con frecuencia resulta difícil, si no imposible, definir el estado normal de un cuerpo o mente humanas. Por consiguiente, el concepto de mejora humana se torna borroso. Por esta razón, los éticos y los científicos sociales se han esforzado en obtener algún criterio más definido. Así, a menudo se ha contrapuesto la mejora a la terapia. En el ámbito médico, en la legislación y en los contratos de seguros "terapia" se emplea como sinónimo de "tratamiento", con el objetivo de remediar un problema de salud, usualmente tras el diagnóstico del mismo. Una patología puede disminuir las capacidades de un individuo por debajo de lo que habitualmente "rendían". En cambio, la mejora supone aquella intervención tecnológica que persigue mejorar una condición del cuerpo o de la mente que se entiende funciona con normalidad o que se encuentra dentro de las capacidades normales de las personas. Así, unas gafas para corregir la miopía están dentro de la terapia, mientras que un visor de visión nocturna constituye una mejora. Un medicamento administrado para tratar la hiperactividad infantil es un caso diferente de la misma sustancia ingerida para mejorar el rendimiento en un examen. ${ }^{2}$

La distinción entre terapia y mejora tiene la ventaja de ser simple y práctica. Desempeña un papel importante en las decisiones relativas a la cobertura de los seguros médicos y, además, puede ser invocada como criterio a la hora de desarrollar políticas públicas en el ámbito de la salud (Malsch y Hvidtfelt-Nielsen, 2010). Sin embargo, debemos ser conscientes de que esta distinción no se aplica con tanta facilidad como se pudiera pensar en las prácticas terapéuticas contemporáneas. La razón de ello reside en el hecho de que las terapias preventivas, de apoyo y de otros tipos, funcionan como tratamientos para evitar problemas de salud futuros y para modificar o mejorar el grado de comodidad del paciente, su bienestar, su aspecto, su esperanza de vida, etc. Pensemos en la cirugía plástica, las vacunas (que mejoran el sistema inmune en ausencia de enfermedad), los métodos anticonceptivos (que previenen un embarazo indeseado) o los procedimientos dentales (que acaso proporcionen una dentadura más bonita y resistente de la que se tenía antes del procedimiento), entre muchos otros. Para un número cada vez mayor de personas, la cirugía y los implantes son la manera que tienen de verse mejor a sí mismos, y por tanto, de mejorar su estado de salud psicológico.

Por estos y otros motivos, se ha formulado un conjunto de objeciones contra todo criterio basado en la distinción entre terapia y mejora en las reflexiones éticas sobre

Los ejemplos provienen de Allhoff et al., (2009). 
la mejora humana. Curiosamente, en algunos puntos se ponen de acuerdo tanto partidarios como detractores de las tecnologías para la mejora humana, extrayendo no obstante consecuencias éticas diametralmente opuestas. En un lado, tenemos a defensores a ultranza de la mejora humana como son los transhumanistas. ${ }^{3}$ En el otro, quienes creen que transitar esa vía va a producir mucho más daño que beneficio a la humanidad, por lo que debe ser evitada a toda costa. El transhumanismo es un movimiento internacional que aboga por el uso extensivo de las tecnologías para la mejora humana apelando, justamente, a motivos éticos: el alivio o la eliminación del sufrimiento físico y mental, la enfermedad, la vejez y hasta la muerte. En definitiva, se trata de luchar contra los aspectos indeseables de la condición humana con las armas tecnológicas con las que la inteligencia nos ha dotado. Por ejemplo, el bioético Julian Savulescu, de la Universidad de Oxford, ha examinado el futuro de los seres humanos como productos de la evolución, centrándose en aspectos tales como —a su juiciosu limitado altruismo, instintos de cooperación y capacidad para tomar suficientemente en cuenta las consecuencias futuras de sus acciones. A partir de este análisis argumenta que la psicología y biología humana no están preparadas para el tipo de sociedad en el que vivimos y que, en consecuencia, o bien modificamos nuestras instituciones políticas y restringimos severamente nuestra tecnología o cambiamos el futuro empleándolas para mejorarnos genéticamente con el fin de evitar nuestra extinción. ${ }^{4}$

Uno de los más conocidos paladines del movimiento transhumanista, el filósofo Nick Bostrom, ${ }^{5}$ critica la distinción entre terapia y mejora porque la considera una petición de principio, es decir, presupone que ya se ha definido lo que constituye un estado normal de salud, que es justamente el problema a debatir. La naturaleza humana "normal" se puede definir desde el nivel de la caracterización de la especie, o desde el punto de vista estadístico del rango de funcionamiento "normal" de los individuos, pero también desde el punto de vista histórico, filosófico o religioso. Evidentemente, las interminables disputas acerca de lo que es la naturaleza humana o la condición humana (por ejemplo, entre esencialistas y antiesencialistas) no facilita el consenso sobre lo que sería un estado psicofísico normal al que contraponer las intervenciones de mejora justificadas o injustificadas, morales o inmorales. Por otro lado, si se incide demasiado en la conveniencia de preservar un estado físico y mental "normal", definido por los medios que fuere - la "normalización" del cuerpo humano- ello podría discriminar negativamente bien a quienes tienen discapacidades, bien a aquellos otros que poseen capacidades por encima de la media, en tanto se paralizaran investigaciones relevantes para esas personas.

En realidad, hace tiempo que iniciamos las acciones de alteración del cuerpo y de la mente con un conjunto de sustancias, materiales y dispositivos incorporados, como son los marcapasos, las prótesis dentales y de cadera, la piel artificial, los implantes de córnea, y tantas otras aplicaciones. Para los transhumanistas, dado que toda la especie humana es por definición "discapacitada"6 y se encuentra necesitada de mejo-

\footnotetext{
3 Su asociación, conocida hasta el año 2008 como la Asociación Transhumanista Mundial (World Transhumanist Association- WTA) , cambió su nombre por "Humanity+". Su página oficial es http://humanityplus. org/

4 Otros argumentos a favor de la mejora humana pueden encontrarse en Savulescu y Bostrom (2009).

5 Véase por ejemplo Bostrom y Roache (2008).

6 En Wolbring (2005) se formula una extensa crítica contra los programas de mejora en relación con el concepto de discapacidad.
} 
ra, sería una cuestión de grado el continuar por esa senda hasta llegar, por decirlo en términos un tanto dramáticos, a transformarnos en verdaderos organismos cibernéticos o "ciborgs".

Por si fuera poco, los avances en el conocimiento del funcionamiento del organismo humano, gracias a la ciencia y a tecnologías convergentes como las nanotecnologías, irán proporcionando tal cantidad de información "individualizada" que esa acumulación de datos sobre las circunstancias psicofísicas específicas de cada persona contribuirá a dificultar las definiciones estándar de salud y de patología, así como los tratamientos generales de la enfermedad (Nanomed Roundtable, 2010).

\section{EL SIGNIFICADO DE LO HUMANO}

En el lado opuesto del transhumanismo, se encuentran quienes ven en los programas tecnológicos de mejora incertidumbres y riesgos terribles para nuestra especie como un todo. Unos inciden en la separación tajante entre terapia y mejora como criterio para evitar los males de esta última (véase, por ejemplo, Ach y Lüttenberg, 2008). Otros en cambio, coinciden con los transhumanistas en que las fronteras entre usos terapéuticos y usos para la mejora son imprecisas cuando se trata de las nuevas tecnologías. Sin embargo, esta constatación les impele a exigir el establecimiento de límites en la investigación y aplicación de determinados procedimientos terapéuticos. La argumentación de estos últimos viene a ser la siguiente. Una vez entrados en la dinámica de la manipulación humana, es inevitable deslizarse desde el deseo de corregir la miopía (u otra enfermedad de la vista) hasta una visión nocturna más potente; desde una predisposición a sufrir anemia a un torrente sanguíneo lleno de células extra para el transporte de oxígeno; de ser demasiado bajo según los criterios del lugar y del momento a alcanzar la altura de los héroes; de tener un hijo con problemas de rendimiento escolar a un "superdotado". En principio, estas mejoras no suponen un riesgo, en tanto haya consenso sobre su neutralidad desde el punto de vista ético, se consideren benignas o incluso poseedoras de un carácter moral especialmente valioso. ¿Pero, qué sucede si existe dicho consenso pero no iguales medios para obtener las mejoras en caso de desearlas? ¿Y qué sucedería si realmente no existiera siquiera un consenso sobre su deseabilidad? Tenemos el caso relativamente reciente del implante coclear. Este implante se emplea para corregir la deficiencia auditiva profunda neurosensorial. A pesar de su éxito y de lo extendido que están, hay miembros de la llamada "cultura sorda" que lo critican, no por motivos técnicos, sino por argumentar que ser sordo no constituye una "discapacidad", un defecto, que supone simplemente otra forma de relacionarse con el mundo, y que los niños pueden emplear el lenguaje de signos alternativamente al hablado. Sin entrar ahora en lo acertado o no de estas reticencias sobre el uso de una tecnología para remediar lo que se suele considerar una disfunción del sentido del oído, podemos recurrir a ejemplos muy conocidos, como es el de quienes rechazan transfusiones de sangre por motivos religiosos.

En otras palabras, ya al día de hoy se plantean reservas morales respecto a un conjunto de actividades de mejora tecnológica que supuestamente sólo tienen una función curativa, protésica o regeneradora. Hasta ahora, estas intervenciones ocasionan algunas polémicas entre los grupos directamente afectados por las mismas. Pero si se extienden los métodos tecnológicos de mejora, se alcanzará gradualmente una situación irreversible para el conjunto de la humanidad. Muchas personas, y no sólo 
las creyentes, se sienten profundamente perturbadas ante la perspectiva de un futuro donde ya no esté del todo claro en qué consiste un ser humano. Argumentan que los ciborgs están bien para las películas, pero que es mejor dejar la condición humana intocada, de manera que podamos reconocernos en la historia de la humanidad y, en definitiva, que se pueda preservar el significado del tipo de condiciones, actividades y valores que nos definirían como tales. Volviendo al tema del deporte, es evidente que si cualquiera puede modificarse de manera que se convierta en un superatleta, entonces el hecho de que pueda batir sus anteriores marcas sin esfuerzo no tendrá el significado de superación que le damos a alguien que entrena duro para mejorar su rendimiento deportivo. Por otro lado, si todos acceden a las mismas mejoras, entonces éstas se anulan y a efectos prácticos es como si no se hubiera mejorado ninguno; ahora bien, con respecto al pasado de la actividad deportiva humana, se habría roto esa cadena de logros que nos conecta con los atletas clásicos, y que es reconocible todavía en el momento presente. Hace pocos años, un atleta surafricano con discapacidad, Oscar Pistorius, protagonizó una viva polémica con las autoridades del olimpismo debido a sus pretensiones de correr en los juegos olímpicos de Pekín 2008. Pistorius, al que se le amputaron las piernas de niño, utilizaba unas prótesis con resortes que le permitían correr a gran velocidad. Algunos pensaron que esto le otorgaba una ventaja injusta sobre los atletas que no utilizaban tal dispositivo, es decir, los atletas "normales". Aquí se daba la paradoja de que un "discapacitado" al final estuviera más capacitado para correr con rapidez que la mayoría de los seres humanos no discapacitados.

Entre las voces que critican los programas de mejora humana a gran escala, podemos mencionar al conocido escritor y activista ambiental Bill McKibben, quien precisamente ha recurrido al ejemplo del deporte, entre otros, para clarificar su posición. Este autor se suma a las voces de los que reclaman que la sociedad detenga ciertos avances cruciales en ingeniería genética, robótica y nanotecnología, dado que ponen en cuestión lo que constituye un ser humano y la capacidad de elección de las generaciones futuras. McKibben nos urge a decir "basta". No de una manera, por expresarlo así, integrista; no se trata de impedir todo tratamiento nuevo ni de dejar de luchar contra la discapacidad. Lo que nos sugiere McKibben es que tomemos consciencia de que, si bien ciertamente tenemos problemas serios, en las sociedades modernas ya vivimos desde hace tiempo razonablemente bien. Tener más no significa por fuerza ser más feliz. Las soluciones aportadas por las nuevas tecnologías vendrán probablemente de la mano de problemas inesperados. En sus fases más avanzadas, estas transformaciones tal vez podrían "destruir el significado de nuestras vidas" (McKibben, 2003: 95). Con esto se oscurecería gran parte del supuesto beneficio que reportarán las tecnologías de mejora: mayor productividad y creatividad, vidas más largas, cuerpos y mentes más fuertes, entre otras. Por no mencionar que la acumulación de esas capacidades no equivale a llevar vidas más felices, lo mismo que al día de hoy quienes más poseen no necesariamente llevan vidas más satisfactorias (Allhoff et al., 2009).

Lo interesante de esta línea de argumentación es que no sólo tiene que ver con un futuro más o menos lejano. Tiene que ver incluso más con el día de hoy. Como señala McKibben, necesitamos una nueva manera de mirar al presente (McKibben, 2003). Diseñaremos el futuro de acuerdo con cómo percibamos la situación actual (individual y colectiva); si consideramos que es aceptable o ya insostenible. 


\section{MEJORAS INCREMENTALES Y MEJORAS RADICALES}

Justamente en el presente surgen otros argumentos en contra de los programas tecnológicos de mejora que estamos evaluando, argumentos menos "metafísicos", si se quiere decir así. Para evaluarlos éticamente, algunos autores (Khushf, 2008; Bruce, 2007) proponen distinguir las mejoras incrementales (o de fase 1) de las mejoras radicales (o de fase 2). Las primeras suponen un cambio de grado en las capacidades humanas, son de tipo discreto, implicando un aumento modesto, con daños y beneficios cuantificables y en todo caso evaluables con los recursos actualmente disponibles. Constituyen ejemplos de este tipo de mejoras incrementales el uso de fármacos para mejorar las funciones cognitivas, la cirugía estética y el dopaje en el deporte. En cambio, las modificaciones radicales suponen cambios de estado permanentes e irreversibles y aceleran su propio desarrollo en una dinámica de autorrefuerzo y mediante la convergencia de varias plataformas tecnológicas. Pueden suponer la producción de capacidades radicalmente nuevas para sus poseedores, como la visión fuera del espectro visible para el ojo humano normal, o nuevas formas de comunicación (entre cerebros) o de desplazamiento. Esto hace que la reflexión ética tradicional, que analiza lo que ya ha sido logrado técnicamente, deba completarse con un enfoque proactivo, de trabajo, en colaboración con los investigadores, los científicos sociales y humanistas, los políticos, los juristas, etc. El objetivo es el de anticiparse a los dilemas éticos que tales innovaciones puedan traer consigo, o incluso el de intentar impedir que tengan lugar si se consideran socialmente indeseables.

En lo que sigue nos centraremos en los aspectos problemáticos de los programas de mejora humana, dando por sentado que las mejoras sobre las que existe el consenso de que vale la pena perseguirlas no requieren una discusión en sí mismas. En cualquier caso, en internet es fácil encontrar documentos que inciden en ese carácter positivo de los programas de mejora humana. ${ }^{7}$

Pues bien, de acuerdo con Allhoff et al. (2009) agruparemos los temas controvertidos en cinco apartados: libertad y autonomía; salud y seguridad; justicia y equidad; disrupción social y dignidad humana.

\section{LIBERTAD Y AUTONOMÍA}

Comenzamos con los usos dudosos de la "ingeniería humana". Hay personas que modifican su cuerpo mediante tatuajes, piercings, implantes y otros procedimientos quirúrgicos, no por necesidades terapéuticas ni por obtener alguna mejora incuestionable, sino tan sólo por gusto (por ejemplo, para parecerse a un animal que les resulta sugerente). Hay padres con sordera que desean tener un niño sordo y para ello piden seleccionar el embrión en un procedimiento de fertilización in vitro. Estas acciones y deseos plantean el problema de hasta qué punto la libertad y autonomía de los individuos debe primar cuando sólo implican la modificación voluntaria de su cuerpo por una mejora únicamente percibida por ellos, o su derecho como padres a seleccionar al hijo que desean. En este último caso es evidente que la salud y el bienestar del niño están en juego, pero no cabe decir lo mismo de modificaciones voluntarias que no cau-

Para un enfoque positivo ya clásico de la convergencia positiva de las tecnologías NBIC (nano, bio, info, cogno) véase Roco y Bainbridge (2003). 
san perjuicios a terceras personas. Este tipo de conflicto es similar al que tiene lugar en multitud de facetas de nuestra vida: interés privado versus interés general; privacidad versus seguridad; libertad de expresión versus difamación, etc. En nuestras sociedades los derechos individuales están limitados por consideraciones de orden mayor o cuando entran en conflicto con otros derechos y obligaciones. Así pues, habría que analizar caso por caso para determinar hasta qué punto existiría libertad para operar la modificación o, en su lugar, sería necesario restringirla o incluso prohibirla. Tal cosa podría suceder si se demostrara que la persona está expuesta a un daño por ser sometida a la intervención de ingeniería humana (un riesgo grave para la vida o la salud, por ejemplo, o bien otros efectos secundarios negativos) (President's Council on Bioethics, 2003).

A esto hay que añadir que las presiones externas o modificaciones realizadas en nuestra psique (mediante drogas, implantes, etc.) pueden limitar muy severamente la libertad real de elección. Como ejemplo espectacular cabe citar los recientes experimentos para modificar los propios juicios morales mediante la aplicación de campos magnéticos en determinadas zonas del cerebro (Young et al. 2010). Experimentos como éstos ponen en cuestión también la protección de la salud, la seguridad y la dignidad humanas.

\section{SAlud y SEguridad}

En los casos de modificación (sea por una mejora percibida o real), la libertad del individuo puede entrar en conflicto con los valores de salud y seguridad. Incluso en el caso de que éste alegue que toma la decisión voluntariamente, se pueden dar casos donde el consentimiento informado no se ha cumplido con todas las garantías, o donde una modificación que en principio sólo afectaba al individuo tenga consecuencias negativas en otros (como el conductor ebrio que tiene un accidente, causando la muerte de otra persona).

Se están desarrollando usos militares como exoesqueletos y trajes de campaña para proporcionar a los soldados mejor camuflaje, mayor fuerza y resistencia, alta protección frente a las heridas y tratamientos de primeros auxilios -además de un conjunto de dispositivos integrados en el tejido o en el cuerpo para las comunicaciones, la mejora de las capacidades perceptivas, etc. ${ }^{8}$ Evidentemente, el aumento de la protección de los soldados de un ejército puede suponer una ventaja desproporcionada sobre el contrario, y fomentar un sentimiento de confianza en la victoria que aumente a su vez los casos de uso de la fuerza para resolver los conflictos políticos entre los países.

\section{JUSTICIA Y EQUIDAD}

El derecho a la mejora es invocado por sus partidarios no únicamente como una elección libre, sino apelando a otros derechos, como el derecho a la vida, la salud, a la

\footnotetext{
8 Un ejemplo de este tipo de investigación es el Institute for Soldier Nanotechnologies o Instituto de Nanotecnologías para el Soldado (http://web.mit.edu/isn) del MIT (Instituto Tecnológico de Massachusetts). Ya es habitual el uso de fármacos entre las tropas norteamericanas (entre otras) como antidepresivos y ansiolíticos para combatir el miedo, disminuir el malestar psicológico y la ansiedad y estimulantes para contrarrestar los efectos ocasionados por la falta de sueño.
} 
felicidad, entre muchos otros. Ahora bien, no es en modo alguno un derecho incuestionable. Más problemático es que traiga aparejado más que un incremento de derechos nuevos, la modificación negativa o el decrecimiento en el cumplimiento de los ya existentes (como el derecho a la igualdad).

Existe el riesgo de que se produzcan espirales o dinámicas competitivas crecientes. Dado que la aplicación de mejoras estaría motivada en muchos casos por el deseo de obtener ventajas competitivas, la capacidad para mantenerse por delante de los demás dependería de una carrera continua para la incorporación de mejoras. Los criterios por los que se juzgan las capacidades y rendimiento humanos se tornarían cada vez más cambiantes e inestables. Esto podría afectar al trato justo e igualitario de todos los ciudadanos.

Las ventajas obtenidas por unos podrían disfrutarse a costa de las desventajas relativas sufridas por quienes no hayan podido mejorarse, ya sea en los deportes, el rendimiento académico, las oportunidades de trabajo o en cualquier otro aspecto de la vida en sociedad. Si los ricos son los primeros en adoptar las tecnologías de mejora, dado que son los que más se las podrán permitir con mayor facilidad (como ocurre con las intervenciones quirúrgicas y tratamientos punteros, en la mayoría de los países), se amplificarían las desigualdades sociales, la distancia entre los que tienen mucho y los que tienen poco o apenas nada. Se sostiene que la nanotecnología traerá consigo una "nanodivisión", creando mayores asimetrías y desigualdades dentro y entre los países (Invernizzi, Foladori y Mclurcan, 2008), semenjante a la "brecha digital"; de manera similar, las aplicaciones nanotecnológicas (o "convergentes") podrían crear una brecha o división injusta entre los seres humanos mejorados y los no mejorados. Aquí se plantea el problema de la distribución justa de los bienes que supone el acceso a esas mejoras (justicia distributiva).

\section{DISRUPCIÓN SOCIAL}

Mejoras relativamente pequeñas, cuando las disfruten pocas personas, pueden tener un gran efecto si se acumulan, como por ejemplo la extensión de los años de vida, que es en principio un gran beneficio para el individuo. Al día de hoy los sistemas de pensiones y las prestaciones sanitarias y de otro tipo se ven amenazadas (o al menos eso se argumenta) debido al hecho de que las personas viven de media muchos años tras su jubilación. La extensión de la vida puede producirse por el desarrollo de tratamientos más efectivos contra enfermedades graves (como el cáncer) o por medio de métodos de rejuvenecimiento del cuerpo y de la mente. Esta extensión de la vida, que se cuenta en una media de unos años (pongamos una década o dos) ya causa problemas sociales, pero ¿qué diríamos de una extensión de cien o de mil años, como a la que aspiran algunos visionarios? ${ }^{9}$

Muchas instituciones y esferas de la vida social podrían verse afectadas por la extensión de los programas de mejora. Los cambios en los deportes serían algo menor comparado con los que hubiera que realizar en el sistema de pensiones, la sanidad, el empleo ${ }^{10}$,

\footnotetext{
9 A los problemas evidentes de sobrepoblación, habría que añadir otro más “filosófico" pero no menos grave: la alteración o pérdida del sentido de la vida, que está indisolublemente ligado (o eso al menos creemos) al envejecimiento y la muerte.

${ }^{10}$ Los mejores candidatos serían aquellos que hubieran adaptado su cuerpo o sus capacidades mentales a los requerimientos del puesto de trabajo.
} 
la privacidad, ${ }^{11}$ las comunicaciones, ${ }^{12}$ la gestión ambiental y de recursos estratégicos, como la energía y el agua. La transformación de una institución social no es necesariamente mala; dependería del tipo de modificación o ajuste requerido. Es de esperar que las mejoras operadas en un conjunto de individuos traigan consigo beneficios considerables a la sociedad. Una mayor productividad, creatividad y rendimiento intelectual reportarían beneficios a la sociedad en su conjunto; la cuestión es si esas ganancias superarán los costes y riesgos aparejados. El principio de precaución (o precautorio) pide ser muy cauteloso en el diseño y ejecución de aquellas políticas públicas donde la incertidumbre y los riesgos potenciales son tan elevados.

Debido a la disparidad de experiencias vividas y a las distintas "formas de vida", la cohesión social podría verse afectada por problemas serios de comunicación (comprensión) entre los mejorados y los no sometidos a mejora, o entre quienes han sido mejorados en aspectos distintos. Además, las normas y valores sociales podrían ir degradándose paulatinamente si los estándares humanos cambian a medida que se extiendan las diferencias por medio de las tecnologías de mejora.

\section{MEJORA Y DIGNIDAD HUMANA}

Existe una disputa sobre el efecto de los implantes basados en tecnologías TICs (Tecnologías de la Información y de la Comunicación) sobre la dignidad del ser humano en relación sobre todo a la integridad del cuerpo. Para unos, contribuyen a promover la dignidad al constituir medios para la restauración de las capacidades humanas dañadas, mientras que para otros la ponen en peligro al alterar de manera conspicua cualidades consideradas básicas para la integridad, autonomía y autoconciencia del ser humano (EGE, 2005). Tales implantes, o bien cualquier dispositivo (interno o externo) de acción sobre la actividad cerebral que en principio tenga usos terapéuticos puede ser empleado para acceder a, modificar y controlar la memoria, las percepciones, los hábitos y los juicios del individuo.

La dignidad de los niños nacidos mediante diseño genético puede verse comprometida por responder a fines distintos de los suyos "intrínsecos", como satisfacer las preferencias de sus padres o de quienes posean el poder para tomar decisiones sobre ellos. Su libertad de elección puede verse coartada (si, por ejemplo, se les predispone genéticamente para desarrollar unas habilidades en lugar de otras).

¿Pueden las tecnologías de mejora poner en peligro el desarrollo moral? La madurez del ser humano, la realización de sus potencialidades, implica para muchas personas el planteamiento de unas metas a las que llegar mediante el esfuerzo y el sacrificio. De modo que si la tecnología permite todo tipo de mejoras sin esfuerzo, entonces se perderán oportunidades clave para que el carácter moral pueda desarrollarse. (President's Council on Bioethics, 2003). En la cultura occidental, al menos, los logros son usualmente atribuidos a una persona como agente individual y responsable de sus actos. Si el rendimiento de una persona ya no puede ser contemplado como el resultado de un agente genuino, entonces la idea de mejora que estamos discutiendo puede reducir nuestro sentido de la individualidad, la responsabilidad y la agencia humana (Ach, 2008).

\footnotetext{
${ }^{11}$ Personas con mejoras sensoriales (oído, vista...) podrían acceder con mayor facilidad a informaciones confidenciales.

${ }^{12}$ Los sistemas de comunicación podrían estar incorporados, literalmente, en el individuo.
} 
Esto lleva otra preocupación aparejada, a saber, si en cualquier caso las tecnologías en cuestión harán que nuestras vidas sean más felices. Si la insatisfacción con lo logrado es constitutiva del ser humano, ninguna tecnología lo conseguirá, por muy espectaculares que sean sus logros - a menos que se recurra a las drogas, el lavado del cerebro y otros procedimientos de muy dudosa moralidad.

El sentido comunal de la vida puede perderse si cada individuo se desarrolla en una línea que lo aleja de los demás. La "buena vida" supone un planteamiento común, al menos parcialmente. La sociabilidad humana podría difuminarse con el paso del tiempo. Los seres humanos somos seres sociales (de momento); nuestra idea de la buena vida, y de su sentido, tiene un aspecto comunitario que quedaría amenazado a medida que las mejoras radicales se extendieran, separándonos probablemente cada vez más los unos de los otros. La solidaridad y la compasión podrían verse en entredicho, más de lo que ya lo están en el con frecuencia despiadado mundo moderno.

\section{REPENSANDO LA ÉTICA ${ }^{13}$}

En una gran medida, nuestra ética depende del tipo de seres que somos y de lo que en cuanto tales consideramos pueden ser la felicidad y la vida buena. Tradicionalmente, los filósofos han basado sus teorías éticas en suposiciones sobre la naturaleza humana. Con los nuevos procedimientos tecnológicos de mejora podemos convertirnos en seres distintos en un grado relevante, y con ello tendríamos que repensar nuestras posiciones éticas básicas. Por ejemplo, ¿seríamos igual de solidarios con otros humanos que difieran sustancialmente de nosotros? A mediano plazo parece plausible pensar que las tecnologías convergentes permitirán la realización de mejoras espectaculares, lo cual torna ese escenario en algo a considerar con la debida seriedad.

Hay un cierto sentimiento de urgencia ética a la hora de decidir qué tecnologías deseamos y cuáles queremos evitar. Se requieren mejores condiciones de acceso a la información de relevancia ética y una acción ética mejor en términos proactivos, es decir, de adelantarse a los acontecimientos y de acompañar las tecnologías de mejora a medida que se conciben y se comienzan a poner en práctica. Esperar a que las aplicaciones tecnológicas ya estén en uso permite que éstas se "atrincheren", de manera que es prácticamente imposible la vuelta atrás, aunque con el tiempo se hayan percibido con mayor claridad los dilemas éticos que presentan. Hasta donde sea posible, hay que hacer el esfuerzo por anticipar las consecuencias claramente indeseables de estas innovaciones, estableciendo políticas que minimicen sus efectos negativos. Existen vacíos en las normas y leyes que deben ser rellenados hasta donde alcanzan el mejor conocimiento técnico y el mejor juicio ético disponibles. La ambivalencia ética que pueden presentar muchas nuevas aplicaciones, la borrosidad terminológica, la falta de percepción social de los problemas, las presiones económicas y otros obstáculos no deben impedir el esfuerzo de desarrollar una completa evaluación ética de cada aplicación y más en general, de justificar una política pública sobre la misma.

Las dificultades pueden enfrentarse mejor con la colaboración más eficaz de los éticos, los científicos, los ingenieros y el resto de actores implicados. Junto a ello, cabe desarrollar análisis éticos que dependan menos de teorías éticas simplificadas, que ofrecen poca guía en situaciones particulares. Esto no significa depender únicamente

\footnotetext{
${ }^{13}$ Esta sección se basa en la discusión contenida en Moor (2005).
} 
de enfoques de coste-beneficio, de una evaluación monetaria que en definitiva ignore o minusvalore los valores éticos.

\section{Conclusiones}

En las páginas precedentes hemos realizado un reconocimiento de los elementos principales que pueblan el paisaje moral de la mejora humana mediante las nuevas tecnologías convergentes. La controversia ética sobre este tema está ganando impulso y complejidad, de modo que no podemos pretender otra cosa que haber ofrecido una primera introducción a la misma.

La controversia viene impulsada por el desarrollo de un impresionante conjunto de aplicaciones tecnológicas en la forma de nuevos materiales, nuevas sustancias, nuevos dispositivos. Tales posibilidades alientan ciertas visiones utópicas (y distópicas) del futuro humano. Algunas de esas visiones y, en todo caso, escenarios a corto plazo más pegados a tierra, nos alertan de la llegada de un conjunto de problemas éticos y sociales que hoy en día apenas se esbozan de manera incipiente. De modo que a fin de que la ética, por decirlo así, no actúe con retraso, vale la pena optar por la prudencia y comenzar una reflexión y debate que nos permita, en su caso, preparar convenientemente la normativa y legislación que se requiera con tiempo suficiente (Allhoff et al., 2009). Como en tantos otros campos de la innovación tecnológica, sugerimos la gran utilidad, si no necesidad, de llevar a cabo una evaluación ética de las tecnologías de mejora humana en colaboración con quienes las desarrollan, una evaluación temprana, "en tiempo real" y continuada (de Cózar, 2009b). Tal evaluación deberá dedicar una atención especial a la pregunta de si las mejoras tecnológicas del cuerpo y de la mente contribuyen realmente a la consecución del ideal de vida buena.

\section{BiBLIOGRAFÍA}

Ach, J. S. 2008. “Improving human performance?”. En J. S. Ach y B. Lüttenberg (eds.) Nanobiotechnology, nanomedicine and human enhancement. Berlín: LIT Verlag: 97-111.

Ach, J. S., y Lüttenberg, B. 2008. Nanobiotechnology, nanomedicine, and human enhancement, Münster: LIT Verlag.

Allhoff, Fritz, Lin, Patrick, Moor, James y Weckert, John. 2009. "Ethics of human enhancement; 25 Questions and Answers", US National Science Foundation http://www. humanenhance.com/

Bostrom, N. y Roache, R. 2008. "Ethical issues in human enhancement”. En J. Ryberg, T. S. Petersen y C. Wolf (eds.) New waves in applied ethics. New York: Palgrave Macmillan.

Bruce, Donald. 2007. Human enhancement? Ethical reflections on emerging nano-biotechnologies; Report of the NanoBio-RAISE expert working group on converging technologies and human functional enhancement, Edinburgh: EdinEthics/NanoBio-RAISE. http://files.nanobio-raise.org/Downloads/NBR-enhancement\%20final.pdf

Coenen, Christopher et al. 2009. Human Enhancement, STOA, European Parliament, Brussels http://www.europarl.europa.eu/stoa/publications/studies/default_en.htm

de Cózar, José Manuel 2009. “Aspectos sociales de las nanotecnologías”, en Varios Autores, ¿Qué es la nanotecnología? Avances, expectativas y riesgos, Donostia, Cátedra Sánchez Mazas, colección poliedro: 95-137. 
de Cózar, José Manuel 2009b. “Gobernar la nanotecnología: un (breve) ensayo de democracia técnica”, en Jorge Riechmann, Ed. Nanomundos, multiconflictos. Una aproximación a las nanotecnologías, Barcelona, Icaria: 93-107.

de Cózar, José Manuel 2010. "La vida en una nanocasa. Efectos socioambientales de las nanotecnologías”, en María Casado, Coordinadora, Bioética y nanotecnología, Pamplona, Civitas Thomson Reuters: 255-269.

European Group on Ethics in Science and New Technologies (EGE) 2005.Ethical Aspects of ICT Implants in the Human Body, Opinion $\mathrm{N}^{\circ} 20$ delivered by the European Group on Ethics in Science and New Technologies to the European Commission, Brussels 16 March http://ec.europa.eu/european_group_ethics/publications/docs/avis20compl_en.pdf

Invernizzi, Noela, Guillermo Foladori \& Donald Maclurcan 2008. "Nanotechnology's Controversial Role for the South", Science Technology Society 13: 123.

Khushf, George 2008. "Stage Two Enhancements" en F. Jotterand Ed. Emerging Conceptual, Ethical and Policy Issues in Bionanotechnology(pp. 203-218), Springer.

Malsch, I. y K. Hvidtfelt-Nielsen. 2010. Nanobioethics. Observatory Nano 2nd Annual Report on Ethical and Societal Aspects of Nanotechnology.

McKibben, B. 2003. Enough: Staying human in an engineered age. Nueva York: Henry Holt.

Moor, James H. 2005. "Why we need better ethics for emerging technologies". Ethics and Information Technology 7: 111-119.

NanoMed Roundtable. 2010. A report on the nanomedicine environment. NanoMed Roundtable www.nanomedroundtable.org

Nordmann, A. 2004. Converging technologies: Shaping the future of the european societies. Bruselas, Bélgica, Comisión Europea.

President's Council on Bioethics. 2003. Beyond therapy: Biotechnology and the pursuit of happiness. Washington, DC: Government Printing Office.

Roco, M. and Bainbridge, W.S. 2003. Converging technologies for improving human performance: Nanotechnology, biotechnology, information technology and cognitive science. Dordrecht: Kluwer Academic Publishers.

Savulescu, J. y N. Bostrom (eds.). 2009. Human enhancement. Oxford University Press.

Wolbring, Gregor. 2005. The triangle of enhancement medicine, disabled people, and the concept of health: a new challenge for HTA, health research, and health policy, HTA Initiative series, \#23, AHFMR Health Technology Assessment Unit, Alberta.

Young, Liane, Joan Albert Camprodonb, Marc Hauserc, Alvaro Pascual-Leoneb y Rebecca Saxe. 2010."Disruption of the right temporoparietal junction withtranscranial magnetic stimulation reduces the role of beliefs in moral judgments", PNAS, abril 13, vol. 107 no. 15 6753-6758. http://www.pnas.org/content/107/15/6753 